\title{
Exploration under Sparsity Constraints
}

\author{
Christoph Manss ${ }^{1}$, Dmitriy Shutin ${ }^{1}$, Alberto Viseras Ruiz ${ }^{1}$, Thomas Wiedemann ${ }^{1}$, Joachim Mueller ${ }^{1}$
}

\begin{abstract}
This paper addresses the problem of designing an efficient exploration strategy for multiple mobile agents. As an exploration strategy, an intelligent waypoint generation is considered, where the trajectory of the agent is governed by the properties of the explored phenomenon. Here it is assumed that the explored field is sparse in it's spatial distribution; consequently, it is assumed that a certain agent's movement trajectory might favor a sparse solution, as contrasted to simple sampling strategies. Specifically, these trajectories lead to an emergence of a structured sensing matrix consisting of shifted sensor impulse responses. Nevertheless some properties of this matrix, such as low mutual coherence, are essential for a successful sparse reconstruction of the phenomenon. Thus, the agents are directed to move so as to favor the desired properties of the sensing matrix, an approach termed sparse exploration. Unfortunately, numerical techniques for optimization of the sensing matrix are intractable. Therefore this paper proposes a number of heuristics, which numerically optimize the measurement locations of the agents so as to favor a sparse solution. Synthetic experiments are performed to demonstrate the effectiveness of the proposed heuristics as compared to simple random walk or regular movement patterns.
\end{abstract}

\section{INTRODUCTION}

In exploring an unknown environment with autonomous multiple mobile agents, or swarms, it is crucial to define a main guiding principle that will help a swarm to $a u$ tonomously, i.e., without a direct human interaction, define a next point of interest. For instance, a planetary exploration can exemplify well such scenarios, where a direct steering of a robot on a remote planet might be difficult. Also, such a principle might be helpful for disaster relief organizations, e.g., if robots should autonomously find people in adversity.

Essentially, exploration can be defined as a collection of samples, or measurements, that provide sufficient information about a metric or a field of interest. Depending on a particular environment, these samples can be collected more efficiently if some structure of the explored phenomenon can be assumed. Obviously, it is possible to sample in systematic fashion, over some sampling grids, which take the Shannon sampling theorem into account. Yet when the structure is known, the exploration can profit from it. One reasonable assumption is that the explored phenomenon consists of a relatively small number of well-localized impulses, i.e., that the process is sparse. In this case the explored field can be reconstructed from fewer measurements, compared to Shannon's sampling theorem, i.e., more efficiently using

${ }^{1}$ All authors are with the Institute of Communications and Navigation of the German Aerospace Center (DLR), Oberpfaffenhofen, 82234 Wessling, Germany, christoph.manss@dlr.de, dmitriy.shutin@dlr.de, alberto.viserasruiz@dlr.de, thomas.wiedemann@dlr.de, joachim.mueller@dlr.de methods of compressive sampling [1]-[3]. Thus, for this exploration problem the application of sparse reconstruction techniques are addressed in this work.

In Compressed Sensing (CS) a measurement sample is represented as a linear combination of the original (and sparse) data. This can be described mathematically as $\mathbf{y}=$ $\boldsymbol{\Phi f}$, where $\mathbf{y}$ are $M$ collected samples, $\boldsymbol{\Phi}$ is the $M \times N$ sampling matrix with $M<<N$ and $\mathbf{f}$ is the original data as a $N \times 1$ vector.

If, then, the data is sparse, i.e. the data mostly consists of zeros, CS guarantees the reconstruction of the non-zero components in $\mathbf{f}$ by means of an $\ell_{1}$-constrained optimization [4], even if the sampling equation is highly underdetermined. However, $\boldsymbol{\Phi}$ plays an important part in the CS strategy and certain properties of it are crucial for reconstruction, i.e. finding $\mathbf{f}$. Thus, we would like to optimize $\boldsymbol{\Phi}$ in a way to achieve better waypoints and trajectories for the exploration process.

The idea is to interpret every row of $\boldsymbol{\Phi}$ as a sensor's Channel Impulse Response (CIR), i.e. a footprint of the sensor, which is dependent on the sampling position. Hence, the sampling positions have to be chosen in a way that optimizes $\boldsymbol{\Phi}$.

Nevertheless, the sampling matrix has been optimized in the literature before and other researchers put effort in the use of CS for exploration, measuring and path planning. Some related work is shown in the following.

To optimize the sampling matrix a gradient of a sampling matrix's Grammian matrix is computed in [5]. The sampling matrix is dense and has values drawn from a Gaussian distribution. This algorithm generates sensing matrices with a lower mutual coherence with a sparsifying transformation (dictionary), which improves the reconstruction with Orthogonal Matching Pursuit (OMP). Similar approaches have been made in [6], where the sampling matrix and a dictionary are optimized jointly by minimizing the mutual coherence. In [7] the coherence of a dense matrix is optimized, also, but with a method involving Lagrange multipliers. In this paper the data is already considered being sparse and, hence, has no need of a sparsification by a dictionary. Also, these mentioned methods can not keep the sensor's CIR structure in each row of $\boldsymbol{\Phi}$, because its Grammian is often optimized towards the identity matrix. Therefore they are not suitable for the intended objective in this paper.

An adaptive and distributed method to optimize $\boldsymbol{\Phi}$ has been proposed in [8]. They use an adapt-then-combine diffusion strategy for evaluating the sampling matrix's gradient of multiple agents. Even so, they assume random sampling matrices with values drawn from a Gaussian distribution and they don't consider a structure in the sampling matrix. 


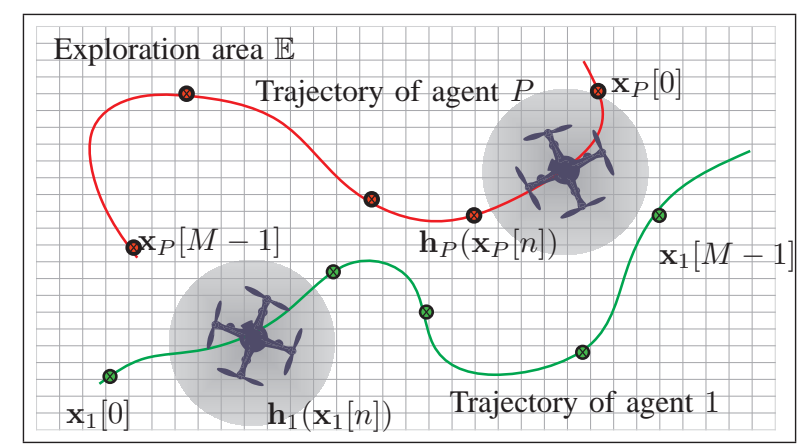

Fig. 1. Measurement model with multiple agents, e.g. UAVs. Each agent has its trajectory with measurement points on it. The environment $\mathbb{E}$ is discretized and the sensor footprint is shown in grey. This footprint is the CIR of the sensor (i.e. h), which is one row of the sampling matrix $\boldsymbol{\Phi}$.

Contrary, [9] proposes a way to construct purely deterministic generated sensing matrices with the use of finite fields. Although, according to the author, the deterministic matrices are always inferior to random matrices, he claims that his approach generates the so far best deterministic constructed sampling matrices. However, these generated matrices are of no use in this paper, because they are not able to take a sensor's CIR into account.

Compressed Sensing for active measuring has been used in [10], where the sampling strategy of a laser scanner is changed to get more performance in the reconstruction by means of $l_{1}$-optimization. They estimate important areas for the scanner to measure and increase the number of samples in such areas.

A path-planning concept for CS is introduced in [11]. They optimize different movement strategies with a particle swarm optimization to reduce the cost of measuring, direction change and motion. Compressed Sensing is then used to reduce the number of measurements, which further minimizes their proposed cost-function. Their approach has less energy consumption and comparable field reconstruction results.

The state of the art shows that CS is useful to save energy and time for exploration purposes, but is still an active field of research. Therefore we propose a new exploration paradigm by means of an adaption of the CS's sampling matrix, which can involve CIRs of sensors, to generate waypoints for exploration purposes. After a problem statement in Section II, different optimization strategies of such structured matrices for exploration purposes are discussed in Section III. The efficiency of those strategies for reconstruction and exploration is evaluated by Monte-Carlo simulations in Section IV.

\section{COMPRESSED SENSING}

Consider now a swarm exploration problem with $P$ independent mobile agents. The goal of the swarm is to cooperatively explore, i.e., sample, a spatially-distributed field $\mathbf{f}$ that is defined over some two-dimensional exploration area $\mathbb{E} \subset \mathbb{R}^{2}$. In the following we will assume that $\mathbb{E}$ is divided into $N$ grid cells, such that $\mathbf{f} \in \mathbb{R}^{N}$ is an $N$-dimensional vector. Each agent performs linear measurements of the field f as follows

$$
y_{i}[n]=\mathbf{h}_{i}\left(\mathbf{x}_{i}[n]\right)^{T} \mathbf{f}+\epsilon_{i}[n],
$$

where $y_{i}[n]$ is the $n$th sample taken by the $i$ th agent, $\mathbf{h}_{i}\left(\mathbf{x}_{i}[n]\right) \in \mathbb{R}^{N}$ is the impulse response of the $i$ th agent's measurement system at location $\mathbf{x}_{i}[n] \in \mathbb{E}$, and $\epsilon_{i}[n]$ is the corresponding measurement noise. By collecting $M$ measurement samples of all $P$ agents in a single vector $\mathbf{y}=\left[y_{1}[0], \ldots, y_{1}[M-1], \ldots, y_{P}[0], \ldots, y_{P}[M-1]\right]^{T}$, we can formulate (1) in a vector form as follows:

$$
\mathbf{y}=\boldsymbol{\Phi}(\mathbf{X}) \mathbf{f}+\boldsymbol{\epsilon}
$$

where $\boldsymbol{\epsilon}=\left[\epsilon_{1}[0], \ldots, \epsilon_{1}[M-1], \ldots, \epsilon_{P}[0], \ldots, \epsilon_{P}[M-\right.$ $1]]^{T}, \mathbf{X}=\left[\mathbf{x}_{1}[0], \ldots, \mathbf{x}_{1}[M-1], \ldots, \mathbf{x}_{P}[0], \ldots, \mathbf{x}_{P}[M-\right.$ 1]], and

$$
\begin{aligned}
\boldsymbol{\Phi}(\mathbf{X})=[ & \mathbf{h}_{1}\left(\mathbf{x}_{1}[0]\right), \ldots, \mathbf{h}_{1}\left(\mathbf{x}_{1}[M-1]\right), \ldots \\
& \left.\ldots, \mathbf{h}_{P}\left(\mathbf{x}_{P}[0]\right), \ldots, \mathbf{h}_{P}\left(\mathbf{x}_{P}[M-1]\right)\right]^{T} .
\end{aligned}
$$

This measuring process is also shown in Fig. 1.

Assuming that $\mathbf{f}$ is sparse, i.e., out of $N$ values only a few are non-zero. ${ }^{1}$ Under this assumption, the theory of CS predicts that $\mathbf{f}$ can be reconstructed more efficiently, in a sense that fewer measurements are needed [2]. Moreover, finding the sparse solution amounts to solving the following $\ell_{1}$-constrained convex optimization problem [1], [2], [12]

$$
\widehat{\mathbf{f}}=\underset{\mathbf{f}}{\arg \min }\|\mathbf{f}\|_{\ell_{1}} \quad \text { s.t. } \quad\|\mathbf{y}-\mathbf{\Phi}(\mathbf{X}) \mathbf{f}\|<\epsilon,
$$

with $\|\boldsymbol{\epsilon}\|=\epsilon$. The success of the optimization (4) in finding a sparse solution hinges upon the properties of the matrix $\boldsymbol{\Phi}(\mathbf{X})$. Specifically, the matrix $\boldsymbol{\Phi}(\mathbf{X})$ has to satisfy a socalled Restricted Isometry Property (RIP) [1]-[3], which implies that

$$
(1-\delta)\|\mathbf{f}\|_{2} \leq\|\mathbf{\Phi}(\mathbf{X}) \mathbf{f}\|_{2} \leq(1+\delta)\|\mathbf{f}\|_{2},
$$

for some small $\delta>0$. In other words, $\boldsymbol{\Phi}(\mathbf{X})$ should behave like a nearly isometric map and this requirement (5) is satisfied also for matrices that have low mutual coherence [7], [13], [14]. Mutual coherence is defined as a maximum correlation between columns of $\boldsymbol{\Phi}(\mathbf{X})$ : a small mutual coherence will also imply RIP, which ensures that (4) recovers a sparse solution.

It can be seen from the model (2) that the properties of the sensing matrix $\boldsymbol{\Phi}(\mathbf{X})$ are essentially determined by the positions of the agents, or, more specifically, by the trajectories of the mobile agents as they move through the exploration area. This permits us formulating a sparse exploration paradigm. Specifically, as sparse exploration we understand an algorithm that computes the movement trajectories of the agents so as to favor a sparse estimate of some (sparse) field $\mathbf{f}$ in the exploration area $\mathbb{E}$ by optimizing the sensing matrix $\boldsymbol{\Phi}(\mathbf{X})$.

\footnotetext{
${ }^{1}$ In a more general setting, we can assume $\mathbf{f}$ to be sparse in some dictionary $\mathbf{D}$, so that $\mathbf{b}=\mathbf{D f}$, with $\mathbf{b}$ being sparse. In this case we can define $\boldsymbol{\Psi}(\mathbf{X})=\boldsymbol{\Phi}(\mathbf{X}) \mathbf{D}$, which will lead to model (2): $\mathbf{y}=\mathbf{\Psi}(\mathbf{X}) \mathbf{f}+\boldsymbol{\epsilon}$. In the following this case will not be considered as it does not impact the methodology we intend to demonstrate later.
} 


\section{SENSING MATRIX OPTIMIZATION}

Sensing matrix optimization is a challenging topic and has been extensively studied in the literature [5], [6], [8], [9]. In our case the direct optimization of $\boldsymbol{\Phi}(\mathbf{X})$ is non-trivial due to the combinatorial nature of the problem [7]: computing the RIP or evaluating mutual coherence would require an exhaustive search over all possible trajectories of all agents. Instead, here we propose to employ several heuristics that optimize the spectral structure of the sensing matrix instead of a direct optimization of the matrix coherence or RIP. In the following the sensing matrix's properties that are more profitable for the estimation problem proposed are discussed in this paper.

First, we would prefer that each new measurement does not decrease the rank of the sensing matrix $\boldsymbol{\Phi}(\mathbf{X})$, which is important for every Basic Pursuit technique [7]. Note that initially, when only a few measurements are available, the sensing matrix is rank-deficient. Thus, our hope is that when the new measurements become available, they will be "orthogonal" to the already available information.

Second, the singular values of the sensing matrix $\mathbf{\Phi}(\mathbf{X})$ should preferably be all equal, or with small variance, to mimic a matrix with low mutual coherence. With these properties in mind we propose the following heuristics.

- linearIndependence: The first heuristic that we propose optimizes the coverage of exploration area for the $k$ th agent as

$$
\begin{aligned}
\widehat{\mathbf{x}}_{k}[n+1] & =\underset{\mathbf{x}_{k} \in \mathbb{E}}{\arg \min } \sum_{p=1}^{P} \sum_{j=0}^{n}\left|\mathbf{h}_{p}\left(\mathbf{x}_{p}[j]\right)^{T} \mathbf{h}_{k}\left(\mathbf{x}_{k}\right)\right|+ \\
& \sum_{q=1}^{k-1}\left|\mathbf{h}_{q}\left(\widehat{\mathbf{x}}_{q}[n+1]\right)^{T} \mathbf{h}_{k}\left(\mathbf{x}_{k}\right)\right|
\end{aligned}
$$

where $n=0, \ldots, M-1$ and $k=1, \ldots, P$. Note that as the full coverage we understand such a measurement strategy under which the agents eventually cover with their sensor footprints the whole exploration area $\mathbb{E}$. Optimization (6) ensures that the correlation between sensor footprints at the existing measurement locations and the next one is minimized. Thus, the row-rank of the matrix grows with each new measurement. As a side-effect, the agents will not take measurements at the same locations. Yet, neither the coherence nor the RIP of the sensing matrix is optimized directly.

- maxDet: Another criterion that we consider aims at optimizing the singular values of the sensing matrix. Again, we do not do it directly, but instead maximize the determinant of the matrix product $\tilde{\boldsymbol{\Phi}}(\mathbf{X})=$ $\boldsymbol{\Phi}(\mathbf{X}) \boldsymbol{\Phi}^{T}(\mathbf{X})$, where we exploit the relationship between the singular values of $\boldsymbol{\Phi}(\mathbf{X})$ and eigenvalues of $\tilde{\boldsymbol{\Phi}}(\mathbf{X})$, which correspond to the RIP in (5) [12]. Formally, the optimization can be stated as follows

$$
\widehat{\mathbf{x}}_{k}[n+1]=\underset{\mathbf{x}_{k} \in \mathbb{E}}{\arg \max } \operatorname{det} \tilde{\boldsymbol{\Phi}}(\mathbf{X}),
$$

where in this case $\tilde{\mathbf{\Phi}}(\mathbf{X})$ is a $(n P+k \times n P+k)$-matrix. Expression (7) ensures that the volume of column space spanned by the sensing matrix grows with each new measurement.

- maxMean, minVar: Another criterion that we propose consists in direct optimization of the eigenvalues, specifically in minimizing their variance and maximizing their mean. Since $\tilde{\boldsymbol{\Phi}}(\mathbf{X})$ is quadratic and positive definite, its eigenvalues $\lambda$ are equal to its squared singular values $\sigma^{2}$. Thus, $\tilde{\boldsymbol{\Phi}}$ 's singular values are optimized to estimate the next location such as

$$
\begin{aligned}
& \widehat{\mathbf{x}}_{k}[n+1]=\underset{\mathbf{x}_{k} \in \mathbb{E}}{\arg \max } \frac{\sum_{j=0}^{n P+k} \sigma_{j}(\mathbf{X})}{n P+k}, \\
& \widehat{\mathbf{x}}_{k}[n+1]=\underset{\mathbf{x}_{k} \in \mathbb{E}}{\arg \min } \frac{\sum_{j=0}^{n P+k}\left(\sigma_{j}(\mathbf{X})-\bar{\sigma}\right)}{n P+k},
\end{aligned}
$$

where $\bar{\sigma}$ is the mean of the singular values of $\tilde{\boldsymbol{\Phi}}$. Also, the number of singular values has to increase after each iteration, i.e., $\operatorname{rank}\{\tilde{\boldsymbol{\Phi}}\} \stackrel{!}{=} n P+k$.

As mentioned before also both criteria can be jointly optimized that $\widehat{\mathbf{x}}_{k}$ fulfills (8) and (9).

\section{A. Algorithm for Waypoint Estimation}

In the following the implementation of the optimization algorithm and, thus, of the way point generation is discussed. The pseudo-code of the method is summarized in the Algorithm 1. At each iteration of the algorithm a next waypoint for the agent is determined based on the above discussed criteria. Essentially, each iteration consists of two parts.

First, a set of possible locations is determined, see Line 3. This functionality is realized in the determineNeighbors () function; here also a check can be implemented to exclude way points that are either obstacles, unreachable due to the motion constraints of the robot or fall outside of the pre-defined search space. Of course, this is only possible if the map is whether estimated before or a-priori known. The search space, over which a list of possible waypoints is determined, can be either local, when only locations in the vicinity of the agent are considered (mainly close to the sensor footprint's border), or global, when the whole environment is taken into account. In the following we will denote these scopes as Local and Global, respectively.

In the second part of the algorithm in Line 5-7 a temporary sensing matrix $\boldsymbol{\Phi}_{\text {temp }}$ is constructed by augmenting $\boldsymbol{\Phi}(\mathbf{X})$ with a new row that models a measurement at a new position - addRow(). A possible new location is taken from the neighbors-array, which has $Q \leq N$ elements depending on the used scope - Local or Global.

Then, depending on the chosen optimization strategy, $\boldsymbol{\Phi}_{\text {temp }}$ is analyzed and if the objective function for a tested position is improved, the position is accepted as the next movement point. The corresponding computations are implemented in the function optimizationStrategy () and realize equations (6), (7), (8), (9) or (8) and (9) together, depending on 
the chosen optimization strategy. Once the solution is found, the sampling matrix $\boldsymbol{\Phi}$ is augmented with the corresponding row, and the new measurement location is stored. As the output of the Algorithm 1 we obtain a set of waypoints and the corresponding sensing matrix $\boldsymbol{\Phi}$.

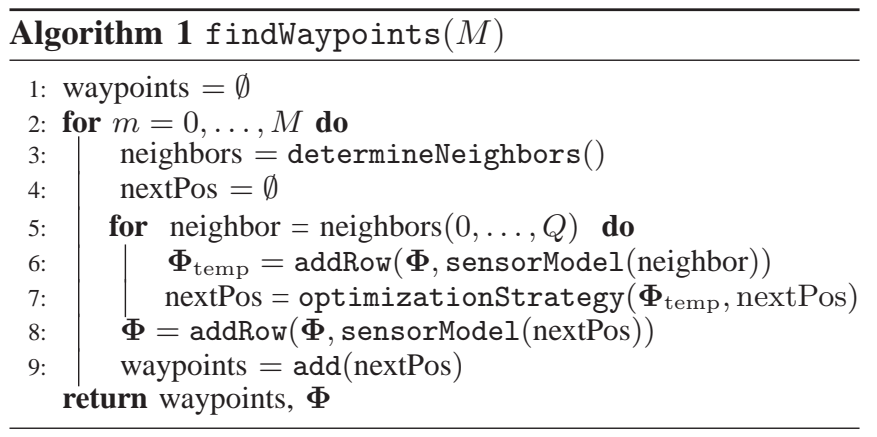

\section{SIMULATION}

\section{A. Description of the simulation}

In this section we will empirically analyze the performance of the sensing matrix optimization strategies discussed above. Therefore we simulate an environment with temperature spots, which have to be estimated in location and intensity with a thermal sensor. For simplicity, we will assume a single agent, i.e., $P=1$, which is simulated as an UAV flying at a height $h$. Although the algorithm is proposed for multiple agents, the result looks similar for one agent, if the estimated waypoints are split and assigned to multiple agents afterwards, assuming that each agent has the same sensor. The exploration area $\mathbb{E}$ can be considered as a square with $30 \times 30$ cells, so that $N=900$. Furthermore, to exclude the impact of the noise on the performance of the estimator, we will assume noise-free measurements, i.e., $\boldsymbol{\epsilon} \equiv \mathbf{0}$. The sensor's footprint is modeled as an indicator function on the interior of the circle with a radius $r=h \sin (\alpha)$, where the sensor has a conical opening with $\alpha$ as opening angle. Furthermore, the interior is weighted by a Gaussian kernel with the width $w$. This results in the sensor's CIR. For all measurements considered here $r \equiv$ const and $w \equiv$ const. In this scenario the sensor footprint has a coverage of about $10 \%$ of the environment, which means 11 measurements cover the environment completely. However, the sensor footprint is circular and the environment rectangular and, thus, overlapping of footprints is unavoidable but required, also.

As references we consider a simple random walk of the agent through the exploration area, and a regular meander pattern. Whereas, the meander counts the number of grid cells in the square environment $\mathbb{E}$ and takes equidistant samples such that as much as possible of the area is covered. The sampling distance of the meander strategy depends on the applied number of measurements.

Each waypoint estimation with reconstruction is applied 500 times in the Global-case and 50 times in the local-case. The location and amplitude of the four non-zero elements in $\mathbb{E}$ and the starting position of the agent is always changed randomly in the beginning of each simulation. Then one of the waypoint estimation strategies is applied and the agent samples at the estimated locations. Afterwards, the resulting measurements and the sensing matrix are used for the environment's reconstruction with the corresponding $\ell_{1}$ constrained reconstruction problem using CVXOPT $^{2}$ library. Subsequently the Normalized Mean Square Error (NMSE) is computed with the original data $\mathbf{f}$ and the estimated data $\widehat{f}$ such as

$$
e=\frac{(\mathbf{f}-\widehat{\mathbf{f}})^{T}(\mathbf{f}-\widehat{\mathbf{f}})}{\mathbf{f}^{T} \mathbf{f}},
$$

which is the optimization strategy's measure of performance and later on shown in Fig. 2 and Fig. 5.

Equally, the mutual coherence is computed to see how the optimization strategies are affected by it.

\section{B. Simulation and Discussion of Results}

As can be seen in Fig. 2 optimization strategy MaxDet (7) result in the lowest NMSE with increasing number of measurements.

If the number of samples is significantly small, MaxMean (8) performs best. The reason for this is that this strategy samples in smaller distances to previous samples and if an impulse is in this sampled area, it can be easily reconstructed. Wheras the MinVar strategy (9) samples with a larger distance to other samples. This leads to many samples close to the border of $\mathbb{E}$. It seems that MaxDet is somehow a tradeoff between those two optimization strategies. Generally, if the number of samples is too small the reconstruction process can not accurately estimate the position of each impulse, which leads to a higher NMSE.

The Randomwalk and Meander strategies are first performing well, but with increasing number of measurements they are outperformed by MaxDet. Also, due to the random nature of the Randomwalk, it is not guaranteed that the whole environment is sensed. The Meander strategy needs a higher number of measurements to obtain a well distanced number of measurements.

The strategy shown in (6) led to bad conditioned matrices with the result that the reconstruction often fails and is therefore not shown in Fig. 2.

A smaller mutual coherence of $\boldsymbol{\Phi}$ does lead to improvements in the reconstruction procedure, as can be seen with MaxDet. However, in the case of RandomWalk and Meander, the reconstruction is still good compared to the other algorithms, although their sampling matrix has a larger mutual coherence.

As an example Fig. 4 shows one sampling grid with 40 measurement locations estimated by the MaxDet strategy. This strategy aims for sampling positions (rectangle) that are somehow located on the border of each footprint (circles). With increasing number of measurements new sampling positions are located in between. This is also the reason why the mutual coherence decreases.

If the view of the optimization strategy is changed to Local, sampling positions near the current positions are

\footnotetext{
${ }^{2}$ http : //cvxopt.org/
} 


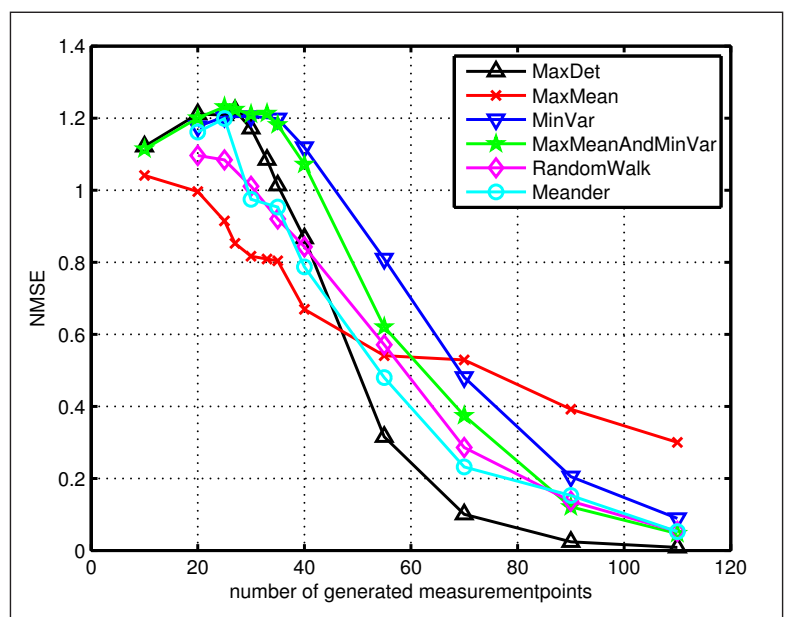

Fig. 2. NMSE dependent on the number of measurements with a globalview. This NMSE is averaged over 500 independent runs, where the impulses positions and the initial positon of the agent are chosen randomly. The NMSE is computed as mentioned in (10).

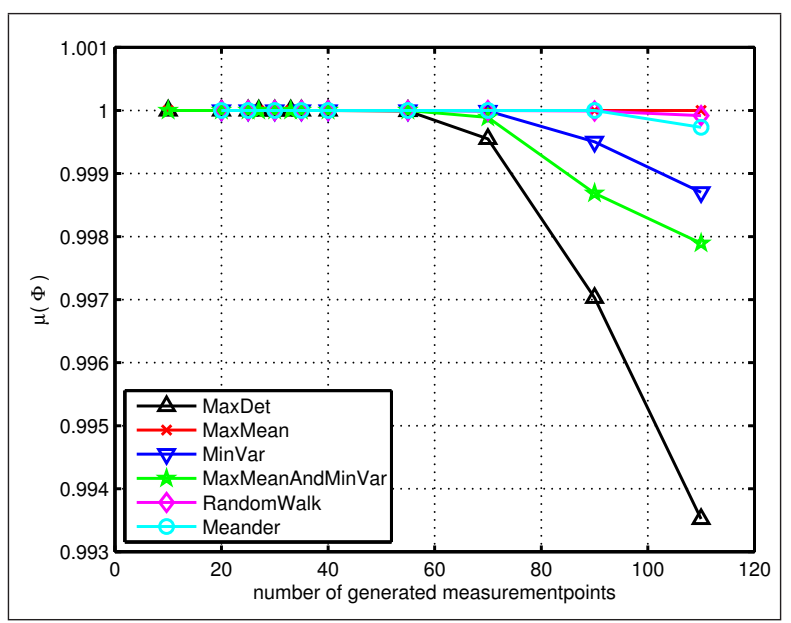

Fig. 3. If the mutual coherence $\mu(\boldsymbol{\Phi})$ decreases, the performance of the reconstruction increases (compare Fig. 2. The mutual coherence depends on the sampling positons and the overlap of the sensor footprints.

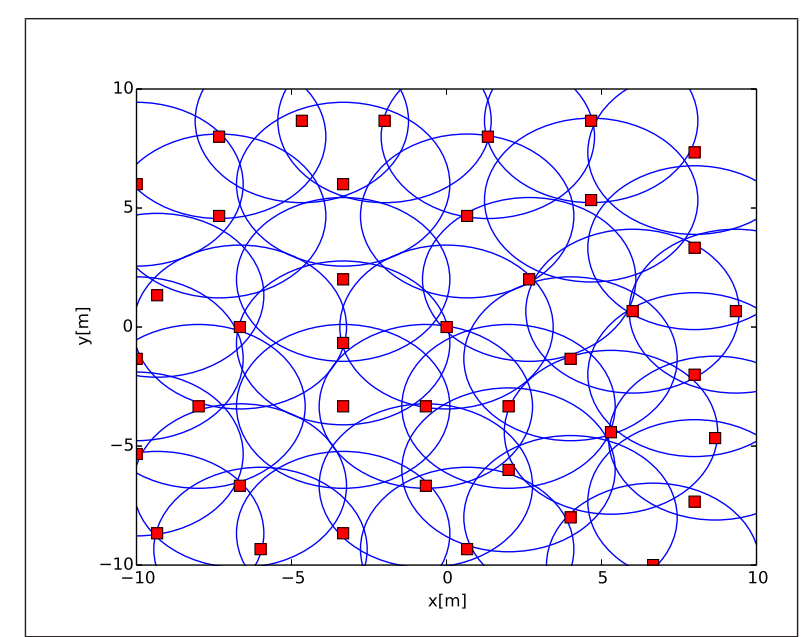

Fig. 4. A trajectory optimized by the MaxDet strategy. The rectangles are the sampling position and the circles indicate the sensor footprint. In total these are 40 measurements. The initial position has been chosen randomly. considered only. The NMSE of the optimization strategies MaxDet, RandomWalk with local-view are shown in Fig. 5. Since the local-view does not offer every position in $\mathbb{E}$, it is inferior to the global-view strategies, but less computational complex. The exploration paradigm is constrained by the agent's position and, thus, makes some bad decisions. This results in a larger NMSE, but still the reconstruction is solved.

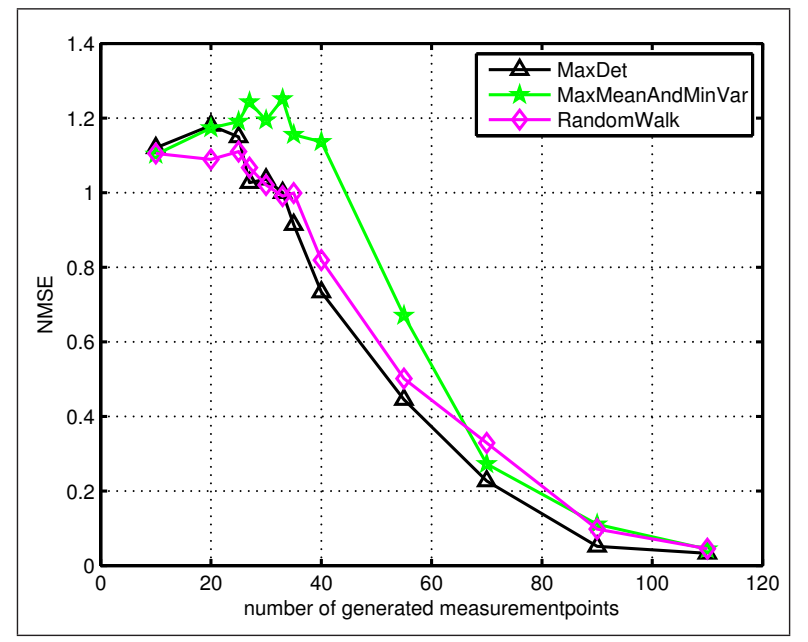

Fig. 5. NMSE dependent on the number of measurements with localview. This NMSE is averaged over 50 independent runs, where the impulses positions and the initial positon of the agent are chosen randomly. Due to the local-view and its constraints the algorithm sometimes makes a bad decision.

\section{CONCLUSION AND OUTLOOK}

In this paper we have shown an exploration paradigm for sparse environments that is based on CS and generates points of interest by optimization of the sampling matrix $\boldsymbol{\Phi}$. Although the optimization of such a matrix is nontrivial, some empiric strategies have been proposed and evaluated. This paper shows, also, how to directly use the sensor's footprint in the sampling matrix, which should make CS more practical for mobile sensing in robotics. These strategies have been tested against each other and the results show that some give good results with respect to the NMSE. Also, due to sparsity and the CS-strategy less than $10 \%$ of all possible measurement points are needed to estimate the whole environment, which in the end saves time and energy.

Our group is looking forward to further investigate such sparse-driven exploration strategies. Therefore, these strategies should be implemented on multiple agents $(P>1)$, i.e. UAVs, to show the performance in reality. Also, these strategies should be enhanced by taking sparsification by means of a dictionary into account. A dictionary should enable these strategies to perform even in non-sparse environments.

\section{REFERENCES}

[1] D. Donoho, "Compressed sensing," Information Theory, IEEE Transactions on, vol. 52, no. 4, pp. 1289-1306, April 2006.

[2] R. G. Baraniuk, "Compressive sensing," Lecture Notes in IEEE Signal Processing Magazine, vol. 24, no. 4, pp. 118-120, Jul. 2007. 
[3] E. Candes and M. Wakin, "An introduction to compressive sampling," Signal Processing Magazine, IEEE, vol. 25, no. 2, pp. 21-30, March 2008.

[4] D. Donoho and X. Huo, "Uncertainty principles and ideal atomic decomposition," Information Theory, IEEE Transactions on, vol. 47, no. 7, pp. 2845-2862, Nov 2001.

[5] V. Abolghasemi, D. Jarchi, and S. Sanei, "A robust approach for optimization of the measurement matrix in compressed sensing," in Cognitive Information Processing (CIP), 2010 2nd International Workshop on, June 2010, pp. 388-392.

[6] O. Endra, "Joint optimization of measurement matrix and sparse dictionary in compressive sensing," in Computer and Communication Engineering (ICCCE), 2012 International Conference on, July 2012, pp. $420-425$.

[7] M. Elad, "Optimized projections for compressed sensing," Signal Processing, IEEE Transactions on, vol. 55, no. 12, pp. 5695 - 5702, Dec 2007.

[8] S. Xu, R. de Lamare, and H. Poor, "Distributed compressed estimation based on compressive sensing," Signal Processing Letters, IEEE, vol. 22, no. 9, pp. 1311-1315, Sept 2015 accepted.

[9] R. A. DeVore, "Deterministic constructions of compressed sensing matrices," Journal of Complexity, vol. 23, no. 46, pp. 918 - 925, 2007, festschrift for the 60th Birthday of Henryk Woniakowski. [Online]. Available: http://www.sciencedirect.com/science/article/pii/S0885064X07000623

[10] S. Huang and J. Tan, "Compressive mobile sensing in robotic mapping," in Intelligent Robots and Systems, 2009. IROS 2009. IEEE/RSJ International Conference on, Oct 2009, pp. 3070-3075.

[11] R. Hummel, S. Poduri, F. Hover, U. Mitra, and G. Sukhatme, "Mission design for compressive sensing with mobile robots," in Robotics and Automation (ICRA), 2011 IEEE International Conference on, May 2011, pp. 2362-2367.

[12] E. Candes, J. Romberg, and T. Tao, "Robust uncertainty principles: exact signal reconstruction from highly incomplete frequency information," Information Theory, IEEE Transactions on, vol. 52, no. 2, pp. 489-509, Feb 2006.

[13] Z. Ben-Haim, Y. Eldar, and M. Elad, "Coherence-based performance guarantees for estimating a sparse vector under random noise," Signal Processing, IEEE Transactions on, vol. 58, no. 10, pp. 5030-5043, Oct 2010.

[14] E. Candes and T. Tao, "Decoding by linear programming," Information Theory, IEEE Transactions on, vol. 51, no. 12, pp. 4203-4215, Dec 2005 . 